\title{
Determination of geometry of heavy ion collisions with forward hadron calorimeter (FHCal) at MPD/NICA
}

\author{
Alexander Ivashkin ${ }^{1 *}$, Dmitry Finogeev ${ }^{1}$, Marina Golubeva ${ }^{1}$, Fedor Guber ${ }^{1}$, \\ Alexander Izvestnyy ${ }^{1}$, Sergey Morozov ${ }^{1,2}$, and Alexander Strizhak ${ }^{1,3}$ \\ ${ }^{1}$ Institute for Nuclear Research of RAS, Moscow, Russia \\ ${ }^{2}$ National Research Nuclear University MEPhI, Moscow, Russia \\ ${ }^{3}$ Moscow Institute of Physics and Technology, Dolgoprudny, Moscow Region, Russia
}

\begin{abstract}
The main purpose of the FHCal is to provide an experimental measurement of a heavy-ion collision centrality (impact parameter) and orientation of its reaction plane. FHCal consists of two identical arms placed at the left/right sides from the beam collision point. Due to the fine modular structure and detection of spectators in both forward/backward regions, the angular resolution of the reaction plane reconstruction is below 30 degrees. Since the heavy fragments escape into beam holes, it is not possible to distinguish the central and peripheral collisions using only the deposited energies in FHCal. The subdivision of the calorimeter into two, inner and outer parts, and the calculation of the energy depositions separately in these calorimeter parts allow one to construct a new observable, the energy asymmetry. Taking the two-dimensional correlation between the energy asymmetry and full energy deposition in the calorimeter, it would be possible to resolve the ambiguity in the centrality determination.
\end{abstract}

\section{Introduction}

The goal of the MPD experiment at NICA is to explore the QCD phase diagram in the region of highly compressed and hot baryonic matter in the region of the collider energy $\sqrt{s_{N N}}=$ $4-11 \mathrm{GeV}$ [1]. The origin of confinement, vacuum properties, and the (chiral) symmetries of the QCD are among the main topics of the MPD experiment studies. The basic strategy of the MPD experiment is to measure a large variety of observables for heavy-ion collisions as a function of the collision energy, centrality, and the system size.

An experimental estimates of global event characteristics in nucleus-nucleus collisions such as the centrality of the collision which is related to the number of participating nucleons and the reaction plane orientation are challenging tasks for any high-energy heavy-ion experiments including the MPD experiment at NICA. In heavy-ion interactions the event-by-event determination of the collision centrality is used to study observables like the collective flow, particle multiplicities and fluctuations which vary strongly with centrality. The collision centrality can be determined either by the multiplicity of produced particles in the participant zone or by measuring the energy carried by non-interacting nucleons (projectile spectators) and detected by the forward hadron calorimeter. The measurement of the number of projectile spectators allows one to estimate the number of the participants and, hence, the impact parameter $b$, which are strongly correlated.

\footnotetext{
*e-mail: ivashkin@inr.ru
} 


\section{FHCal in MPD experimental setup}

The MPD experiment has a close to $4 \pi$ acceptance and is designed to detect charged hadrons, electrons and photons produced in heavy-ion collisions in the energy range and high luminosities of the NICA collider. The side view of the MPD experiment is shown in Fig. 1, left. It includes the superconducting solenoid, Time-Projection Chamber (TPC), Time-Of-Flight (TOF) detector, Electromagnetic Calorimeter (ECal), Forward Hadron Calorimeter (FHCal) and Fast Forward Detector (FD). The main detector components for particle tracking and identification are the TPC and TOF. At the design luminosity, the expected event rate in the MPD is about $6 \mathrm{kHz}$. The total charged particle multiplicity is around 1000 for the most central $A u+A u$ collisions at the top NICA energy of $\sqrt{s_{\mathrm{NN}}}=11 \mathrm{GeV}$. The detector design has a very low material budget to allow for reconstruction of the typical particle transverse momentum below $500 \mathrm{MeV} / c$ expected at NICA energies.

The FHCal $[2,3]$ is designed to determine the collision centrality and the orientation of the reaction plane for the collective flow studies. The event-by-event determination of these quantities is of crucial importance for the analysis of many physics observables. The detector will measure the energy of non-interacting nucleons and fragments (spectators) in nucleusnucleus collisions. The FHCal consists of two hadron calorimeters with 44 modules each ( Fig. 1, right) suited symmetrically from the interaction point. It is a fully compensating modular lead-scintillator calorimeter with high and uniform energy resolution. Each individual module consists of 42 lead/scintillator layers with a surface of $15 \times 15 \mathrm{~cm}^{2}$. The scintillation light is read out via wavelength shifting (WLS) fibers by silicon photomultipliers (SiPM).
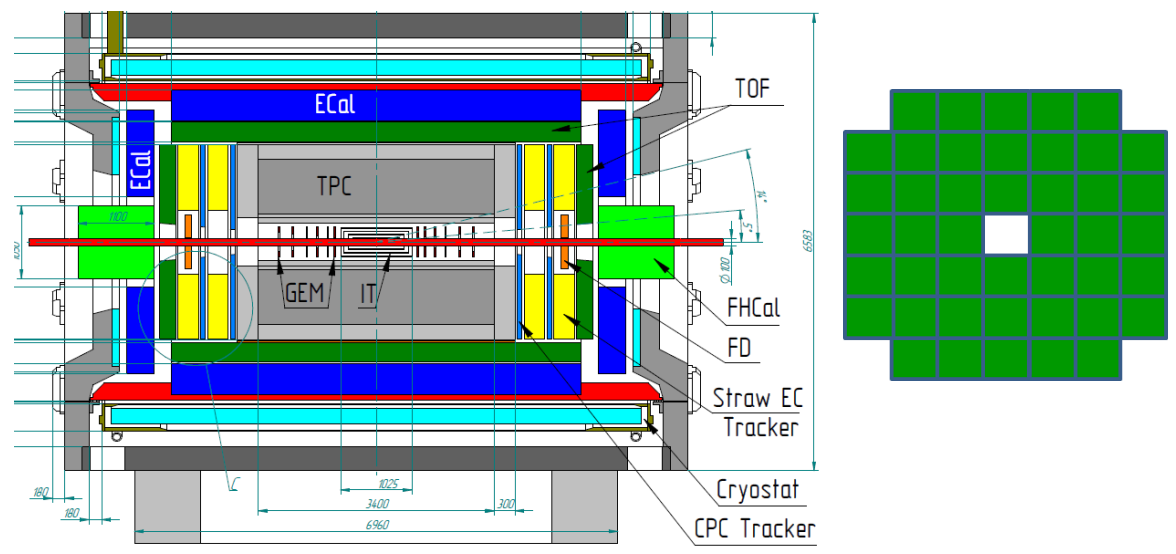

Figure 1. Left - the side view of the MPD experiment. Right - the modular structure of FHCal

\section{Event plane method and reaction plane resolution}

The reaction plane orientation determined by the impact parameter and the beam direction is not known experimentally. To estimate the reaction plane orientation, it is common to use the azimuthal asymmetry of particle production in the plane transverse to the beam direction. Further, the azimuthal angle of the reaction plane in the laboratory frame is denoted as $\Psi_{R P}$.

Due to the momentum transfer between participants and spectators, the spectators (collision fragments) are deflected in the course of the collision. For non-central collisions, the 
asymmetry of the initial energy density in the transverse plane is aligned in the reaction plane, and the spectator deflection direction is correlated with the impact parameter direction. The plane determined by the directions of the beam and spectator deflection (spectator plane) can be used as an estimate of the reaction plane.

The spectator deflection is experimentally accessible. It can be estimated event-by-event by using the transverse segmentation and energy deposition in each of the FHCal modules. In that respect, the FHCal is a unique MPD detector which provides information about spectator deflection in the reaction plane. The estimated azimuthal angle of the spectator plane is called the event plane angle, $\Psi_{E P}$.

The finite number of fragments and the fluctuation of the particle multiplicity from one collision to another at the fixed impact parameter orientation result in a difference between the event plane and the reaction plane orientation. This difference is usually quantified in terms of the event plane resolution (a Gaussian width of the $\left(\Psi_{R P}-\Psi_{E P}\right)$ distribution).

Below we demonstrate the simulated performance of the FHCal for the event plane determination. As a $A u+A u$ collisions generator, the LA-QGSM model [4] is used. The event plane angle is calculated from the energy deposition in a given module of the FHCal by constructing the so-called flow $Q$-vector [5] (two-dimensional vector in the transverse to the beam plane):

$$
\vec{Q}=\left(Q_{x}, Q_{y}\right)=\left(\sum w_{i} \sin \left(\varphi_{i}\right), \sum w_{i} \cos \left(\varphi_{i}\right)\right)
$$

Here $\varphi_{i}$ is the azimuthal angle of the center in the transverse plane of the $i$-th FHCal module, $w_{i}$ is the weight which is used to improve sensitivity of the event plane to the reaction plane. The weight $w_{i}$ was chosen to be the energy in a given module $i$. The event plane angle $\Psi_{E P}$ can be calculated from the $Q$-vector components as:

$$
\Psi_{E P}^{L(R)}=\arctan \left(\frac{\sum E_{i} \sin \varphi_{i}}{\sum E_{i} \cos \varphi_{i}}\right), \sin \varphi_{i}=\frac{y_{i}}{\sqrt{y_{i}^{2}+x_{i}^{2}}}, \cos \varphi_{i}=\frac{x_{i}}{\sqrt{y_{i}^{2}+x_{i}^{2}}} .
$$

Here $E_{i}, x_{i}$ and $y_{i}$ are the energy and coordinates of the $i$-th module, respectively. Index $L(R)$ denotes the left (right) detectors of FHCal.
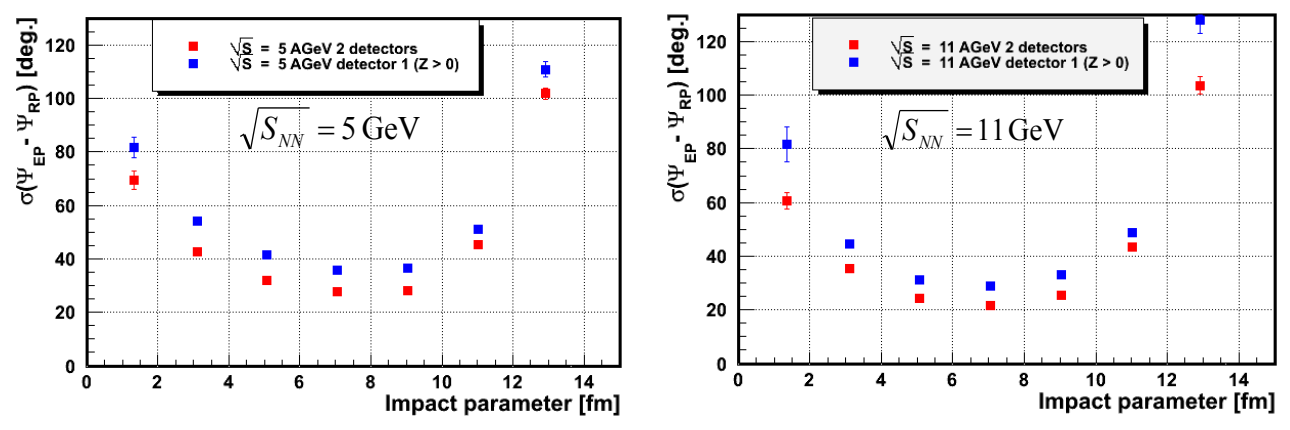

Figure 2. The difference between the reconstructed event plane and true reaction plane for the beam energies $\sqrt{s_{N N}}=5$ (left panel) and $\sqrt{s_{N N}}=11 \mathrm{GeV}$ (right panel). Blue (red) dots correspond to one arm (two arms) of FHCal configuration

The resulting event plane angle $\Psi_{E P}$ is a weighted sum of the $\Psi_{E P}^{L(R)}$. The difference between the reconstructed event plane and true reaction plane reflects the angular resolution of the event plane and is shown in Fig. 2 for the beam energies $\sqrt{s_{N N}}=5$ and $\sqrt{s_{N N}}=$ 
$11 \mathrm{GeV}$. As seen, in both cases the resolution is about 30 degree and it is slightly better for the highest energy.

\section{Measurement of centrality}

The magnitude of the impact parameter, $b$, or the number of participating nucleons for a given heavy ion collision are not known experimentally. Instead, the multiplicity of the produced particles in the overlap zone of the nuclei is used as an experimental proxy of the $b$ value. Since the $b$ value and particle multiplicity are correlated only on the average, the measured multiplicity can be only used to estimate the average impact parameter value and its spread for a given multiplicity (centrality) class of events. In practice, all events are sorted in centrality classes. The most central class is the collisions with the highest multiplicity of the produced particles and the peripheral one is with the lowest multiplicity.

The multiplicity of the spectators (collision fragments) can be also used for collision centrality determination. Spectators provide an independent way to determine centrality which is important for physics studies such as event-by-event fluctuations of various physics observables. In the case of spectator measurements, the most central events correspond to a low spectator multiplicity (or a small energy deposition in the FHCal), while peripheral events result in a large amount of spectators (and typically in a large energy deposition in the FHCal). Fig. 3 shows the impact parameter dependence of the energy deposited in the FHCal. Here the heavy-ion collisions have been also simulated with LA-QGSM model.
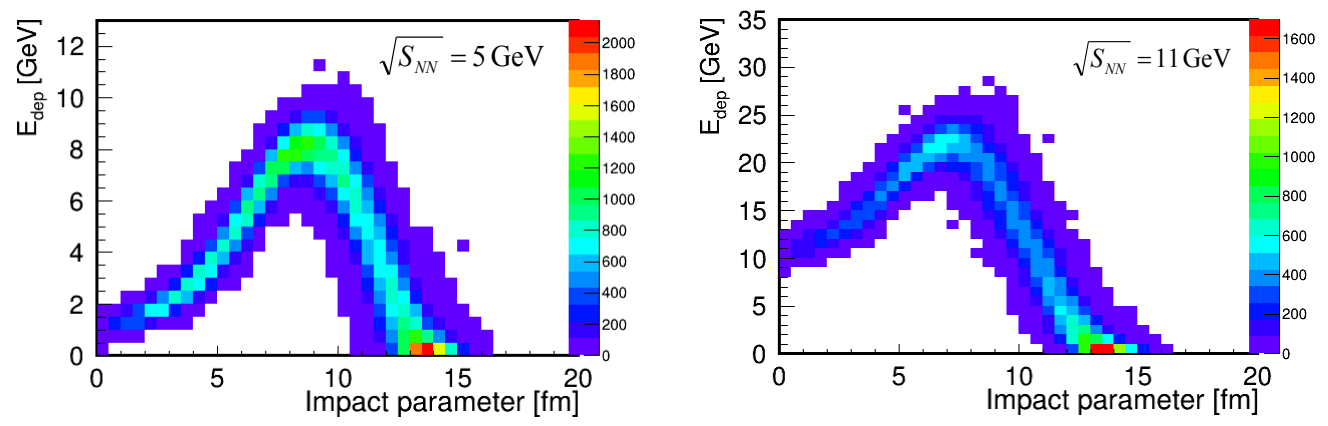

Figure 3. The dependence of energy deposition in FHCal on the centrality for the $A u+A u$ collisions with energy $\sqrt{s_{N N}}=5 \mathrm{GeV}$ (left) and $\sqrt{s_{N N}}=11 \mathrm{GeV}$ (right)

Due to the loss of fragments in the FHCal beam hole the correlation is distorted for peripheral events. As a result, it is not possible to distinguish central and peripheral collisions using FHCal information alone. The energy deposition in the FHCal alone cannot fix the ambiguity in the central and peripheral events. The information about the transverse profile of the energy depositions in FHCal can be used. Clearly, that the number of the fragments which hit the FHCal near the beam axis depends on the impact parameter. Also, the recoil momenta of free spectators (protons and neutrons) and, respectively, their scattering angles depend on the centrality of the collision. As a sequence, the energy deposition in the central and outer regions of FHCal must depend on the centrality. From the energy deposition in inner (outer) modules of the FHCal $E_{\text {in }}\left(E_{\text {out }}\right)$ as illustrated in Fig. 4 (left) one can calculate the asymmetry, $A_{E}$ :

$$
A_{E}=\frac{E_{\text {in }}-E_{\text {out }}}{E_{\text {in }}+E_{\text {out }}}
$$


where $E_{\text {in }}$ and $E_{\text {out }}$ are energy depositions in the inner and outer FHCal modules, respectively.
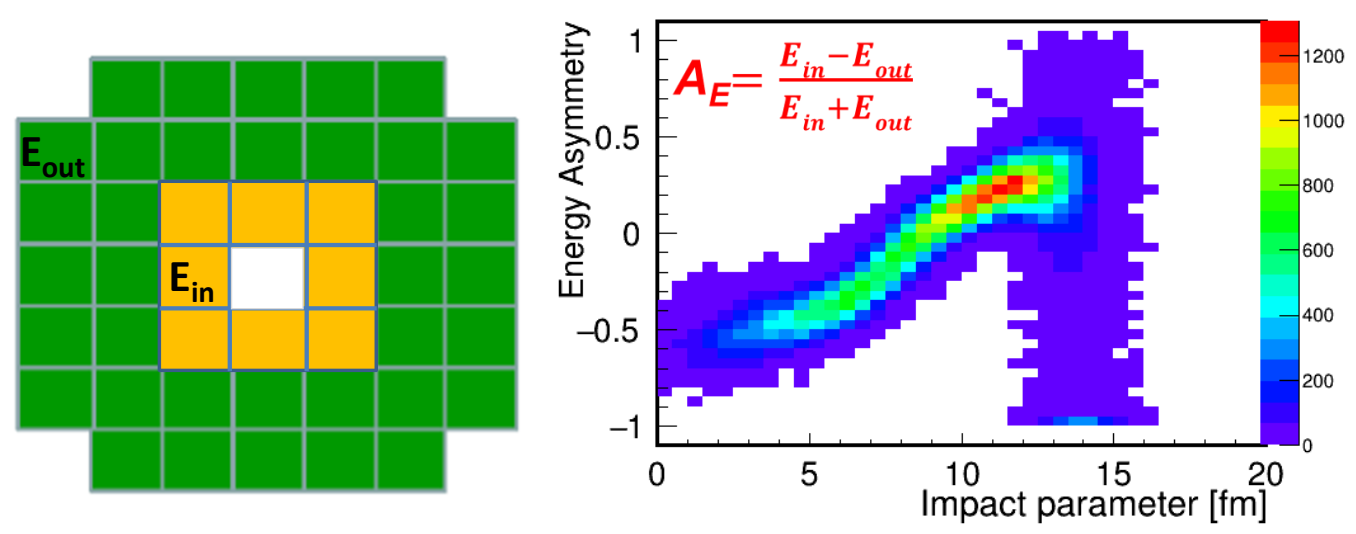

Figure 4. Left - parts of the FHCal used for the calculation of the energy asymmetry. Rightdependence of the energy asymmetry, $A_{E}$ on impact parameter in FHCal for $A u+A u$ collisions at $\sqrt{s_{N N}}=5 \mathrm{GeV}$ simulated with the LA-QGSM model

Fig. 4 (right) shows the dependence of the energy asymmetry $A_{E}$ on the impact parameter for $\mathrm{Au}+\mathrm{Au}$ collision at $\sqrt{s_{N N}}=5 \mathrm{GeV}$. One can see, that both, energy deposition in FHCal and asymmetry dependences might be used to determine the centralities. The correlation between the FHCal energy and asymmetry $A_{E}$ (Fig. 5) avoids the ambiguity and can be used to differentiate the central and peripheral events. The negative (positive) values of the $A_{E}$ correspond to the central (peripheral) events, respectively.
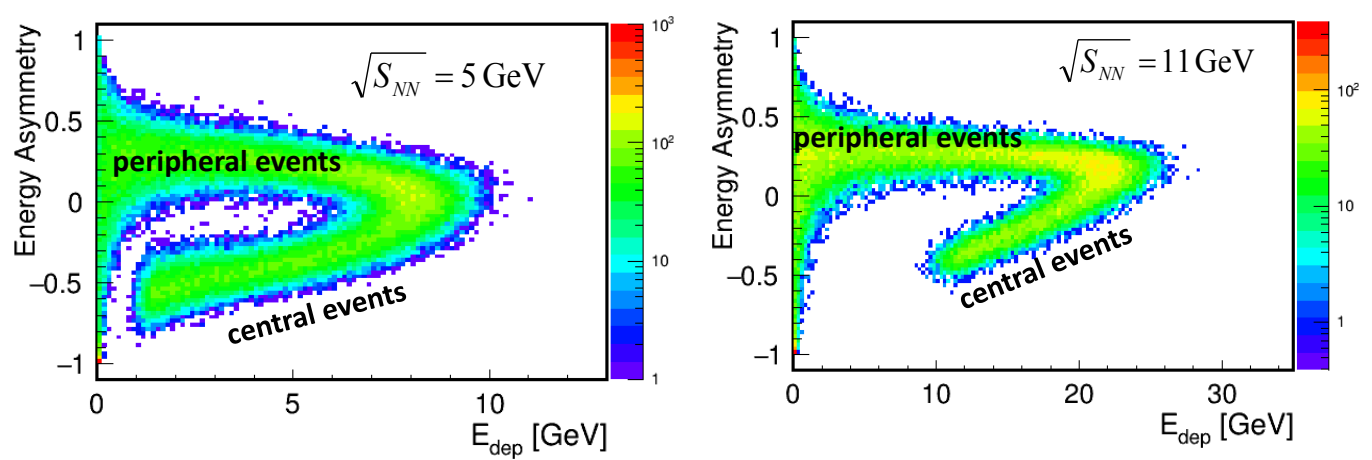

Figure 5. Correlation between the energy asymmetry, $A_{E}$, and total energy deposition, $E_{d e p}$ in FHCal for $A u+A u$ collisions at $\sqrt{s_{N N}}=5$ (left) and $11 \mathrm{GeV}$ (right) simulated with the LA-QGSM model

The corresponding impact parameter resolution is presented in Fig. 6. For beam energy $\sqrt{s_{N N}}=5 \mathrm{GeV}$ blue (red) symbols correspond to the negative (positive) values of the $A_{E}$. The situation is slightly different for $\sqrt{s_{N N}}=11 \mathrm{GeV}$. Here additional region with the $0.1<A_{E}<$ 0.2 is introduced with the corresponding green symbols, see Fig. 6, right. More complicated two-dimensional analysis can be applied to improve the centrality selection. 

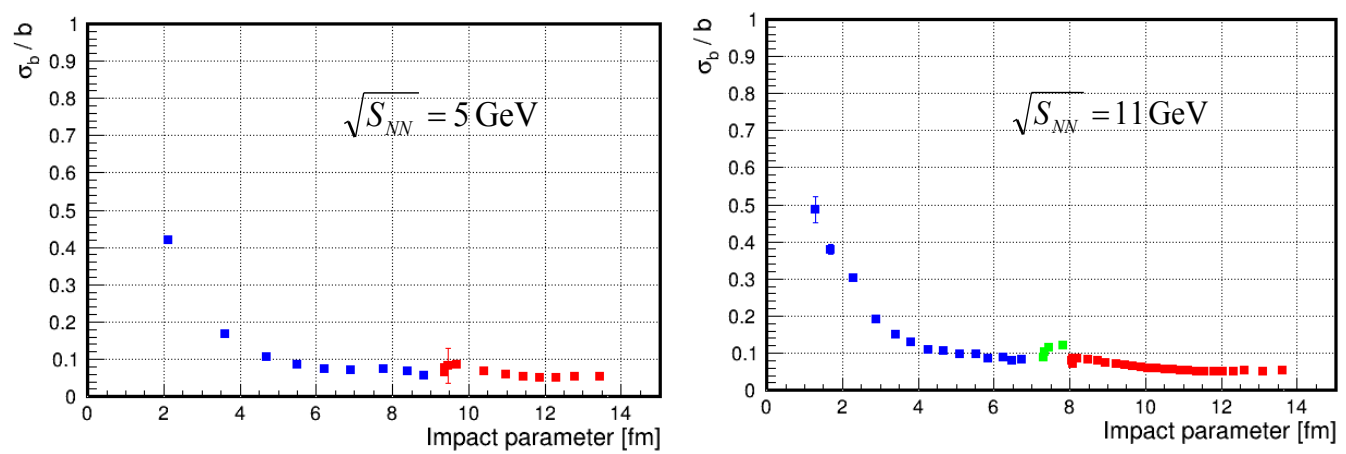

Figure 6. Impact parameter resolution as a function of centrality taken in different energy deposition bins and in different regions of energy asymmetry. For $\sqrt{s_{N N}}=5 \mathrm{GeV}$ (left panel) the centrality bins with negative (positive) $A_{E}$ values are indicated by blue (red) symbols, respectively. For $\sqrt{s_{N N}}=$ $11 \mathrm{GeV}$ the centrality bins with $A_{E}<0.1$ are indicated by blue symbols, with the $0.1<A_{E}<0.2$ - by green symbols and with $A_{E}>0.2$ - by red symbols

One can see, that the resolution of the impact parameters for the mid-central and peripheral events is around or below $10 \%$ in all the range of NICA energies.

Acknowledgements. This work was supported by MPD/NICA collaboration. This work was supported by the Russian Foundation for Basic Research Grant No. 18-02-40065.

\section{References}

[1] A. N. Sissakian and A. S. Sorin, J. Phys. G 36, 064069 (2009)

[2] M. Golubeba et al., J. Phys.: Conf. Ser. 798(1), 012074 (2017)

[3] D. Finogeev et al., KnE Energy and Physics 3, 149 (2018)

[4] S. G. Mashnik et al., arxiv:0805.0751 [nucl-th] (2008)

[5] Arthur M. Poskanzer and S. A. Voloshin, Phys.Rev. C 58, 1671-1678 (1998) 